\title{
Nontrivial symmetric solution of a nonlinear second-order three-point boundary value problem
}

\author{
Yongping Sun
}




\title{
NONTRIVIAL SYMMETRIC SOLUTION OF A NONLINEAR SECOND-ORDER THREE-POINT BOUNDARY VALUE PROBLEM
}

\author{
YONGPING SUN
}

Received 13 January, 2006

\begin{abstract}
In this paper, we study the existence of nontrivial symmetric solution for the secondorder three-point boundary value problem for a function $f:[0,1] \times \mathbb{R} \rightarrow \mathbb{R}$ which is continuous and $f(t, \cdot)$ is symmetric on $[0,1]$. We shall formulate conditions on $f$ which guarantee the existence of nontrivial symmetric solution. As an application, we also give some examples to demonstrate our results.
\end{abstract}

2000 Mathematics Subject Classification: 34B10, 34B15

Keywords: three-point boundary value problem, nonlinear alternative of Leray-Schauder, nontrivial symmetric solution

\section{INTRODUCTION}

In this paper, we study the existence of nontrivial symmetric solution for the threepoint boundary value problem

$$
\begin{gathered}
u^{\prime \prime}(t)+f(t, u(t))=0, \quad 0<t<1, \\
u(0)=u(1)=\alpha u(\eta),
\end{gathered}
$$

where $\eta \in(0,1), \alpha \in \mathbb{R}, f \in C([0,1] \times \mathbb{R}, \mathbb{R}), f(\cdot, x)$ is symmetric on $[0,1]$ for every $x \in \mathbb{R}$, and $\mathbb{R}=(-\infty,+\infty)$.

The three-point boundary value problems for ordinary differential equations arise in a variety of different areas of applied mathematics and physics. For example, the vibrations of a guy wire of a uniform cross-section and composed of three parts of different densities can be set up as a three-point boundary value problem; also many problems in the theory of elastic stability can be handled as a multi-point problem. Many authors studied nonlinear three-point or multi-point boundary value problems and many excellent results have been established. For detail, we refer the reader to $[2-9,11-17,19]$ for some recent results of nonlinear three-point boundary value problems. But the problems of the existence of symmetric positive solutions to the

Research supported by Grant No. Y605144 of NSFZPC and Grant No. 20051897 of the Zhejiang Provincial Department of Education of China. 
second-order three-point boundary value problems has received very little attention in the literature to the best of the author's knowledge. Very recently, Kosmatov [10] studied the existence of triple symmetric solutions for a multi-point boundary value problem by Leggett-Williams fixed point theorem. In [18], we studied the existence of symmetric positive for boundary value problem (1.1), (1.2) by Schauder's fixed point theorem. Motivated by the above mentioned papers, in this paper, we shall study the existence of nontrivial symmetric solution for the boundary value problem (1.1), (1.2) and establish some simple criteria of this problem.

The paper is organized as follows. In Section 2, we obtain some existence results for nontrivial symmetric solution of the boundary value problem (1.1), (1.2). In Section 3, as an application, we give some examples to illustrate the results we obtained. The key tool in our approach is the following Leray-Schauder nonlinear alternative, see for example [1].

Theorem 1.1. Let $E$ be Banach space and $\Omega$ be a bounded open subset of $E$, $0 \in \Omega, T: \bar{\Omega} \rightarrow E$ be a completely continuous operator. Then, either there exist $x \in \partial \Omega$ and $\lambda>1$ such that $T(x)=\lambda x$, or there exists a fixed point $x^{*} \in \bar{\Omega}$.

\section{MAIN RESUltS}

Let $E=C([0,1])$ be a Banach space equipped with norm $\|y\|=\sup _{t \in[0,1]}|y(t)|$ for any $y \in E$. A solution $u(t)$ of the boundary value problem (1.1),(1.2) is called a nontrivial symmetric solution if $u(t) \not \equiv 0$ and $u(t)=u(1-t)$ for all $t \in[0,1]$. To state and prove the main results of this paper, we first give some lemmas.

Lemma 2.1. Let $h \in C([0,1]), \alpha \neq 1, \eta \in(0,1)$, then the three-point boundary value problem

$$
\begin{gathered}
u^{\prime \prime}+h(t)=0, \quad 0<t<1, \\
u(0)=u(1)=\alpha u(\eta)
\end{gathered}
$$

has a unique solution

$$
u(t)=\int_{0}^{1} G(t, s) h(s) d s+\frac{\alpha}{1-\alpha} \int_{0}^{1} G(\eta, s) d s,
$$

where

$$
G(x, y)= \begin{cases}x(1-y) & \text { for } 0 \leq x \leq y \leq 1, \\ y(1-x) & \text { for } 0 \leq y \leq x \leq 1 .\end{cases}
$$

Proof. From (2.1) we have $u^{\prime \prime}(t)=-h(t)$. For $t \in[0,1]$, integrating from 0 to $t$, we get

$$
u^{\prime}(t)=-\int_{0}^{t} h(s) d s+B
$$


For $t \in[0,1]$, integrating from 0 to $t$ yields $u(t)=-\int_{0}^{t}\left(\int_{0}^{x} h(s) d s\right) d x+B t+A$, i.e.,

$$
u(t)=-\int_{0}^{t}(t-s) h(s) d s+B t+A .
$$

Thus, $u(0)=A$ and

$$
\begin{aligned}
& u(1)=-\int_{0}^{1}(1-s) h(s) d s+B+A, \\
& u(\eta)=-\int_{0}^{\eta}(\eta-s) h(s) d s+\eta B+A .
\end{aligned}
$$

Combining with (2.2) we conclude that

$$
\begin{aligned}
& B=\int_{0}^{1}(1-s) h(s) d s, \\
& A=\frac{\alpha \eta}{1-\alpha} \int_{0}^{1}(1-s) h(s) d s-\frac{\alpha}{1-\alpha} \int_{0}^{\eta}(\eta-s) h(s) d s .
\end{aligned}
$$

Therefore, the three-point boundary value problem (2.1), (2.2) has a unique solution

$$
\begin{aligned}
u(t)= & -\int_{0}^{t}(t-s) h(s) d s+t \int_{0}^{1}(1-s) h(s) d s \\
& +\frac{\alpha \eta}{1-\alpha} \int_{0}^{1}(1-s) h(s) d s-\frac{\alpha}{1-\alpha} \int_{0}^{\eta}(\eta-s) h(s) d s \\
= & \int_{0}^{1} G(t, s) h(s) d s+\frac{\alpha}{1-\alpha} \int_{0}^{1} G(\eta, s) d s .
\end{aligned}
$$

This completes the proof.

The following lemma is obvious.

Lemma 2.2. For any $x, y \in[0,1]$, we have

(1) $G(x, y)=G(1-x, 1-y)$,

(2) $G(x, y) \leq G(y, y)=y(1-y) \leq \frac{1}{4}$.

Lemma 2.3. Let $\alpha \neq 1, \eta \in(0,1)$, and $h \in C([0,1])$ be symmetric on $[0,1]$. Then the unique solution $u(t)$ of the boundary value problem (2.1), (2.2) is symmetric on $[0,1]$.

Proof. From (2.3) we have

$$
u(t)=\int_{0}^{1} G(t, s) h(s) d s+\frac{\alpha}{1-\alpha} \int_{0}^{1} G(\eta, s) h(s) d s .
$$


Therefore, for any $t \in[0,1]$, by Lemma 2.2 we get

$$
\begin{aligned}
u(1-t) & =\int_{0}^{1} G(1-t, s) h(s) d s+\frac{\alpha}{1-\alpha} \int_{0}^{1} G(\eta, s) h(s) d s \\
& =\int_{1}^{0} G(1-t, 1-s) h(1-s) d(1-s)+\frac{\alpha}{1-\alpha} \int_{0}^{1} G(\eta, s) h(s) d s \\
& =\int_{0}^{1} G(t, s) h(s) d s+\frac{\alpha}{1-\alpha} \int_{0}^{1} G(\eta, s) h(s) d s \\
& =u(t) .
\end{aligned}
$$

i. e., the solution $u(t)$ is symmetric on $[0,1]$.

Define an integral operator $T: E \rightarrow E$ by the formula

$$
T u(t)=\int_{0}^{1} G(t, s) f(s, u(s)) d s+\frac{\alpha}{1-\alpha} \int_{0}^{1} G(\eta, s) f(s, u(s)) d s .
$$

By Lemma 2.1, the boundary value problem (2.1), (2.2) has a solution $u=u(t)$ if and only if $u$ is a fixed point of the operator $T$ defined by (2.4). So we only need to seek a fixed point of $T$ in $E$. By the Ascoli-Arzela theorem, we can prove that $T$ is a completely continuous operator.

Now we present and prove our main results.

Theorem 2.1. Suppose that $f \in C([0,1] \times \mathbb{R}, \mathbb{R}), f(t, 0) \not \equiv 0 \alpha \neq 1$, and there exist two non-negative symmetric functions $a, b \in L^{1}[0,1]$ such that

$$
|f(t, x)| \leq a(t)|x|+b(t) \quad \text { for a.e. } t \in[0,1] \text { and all } x \in \mathbb{R}
$$

and

$$
\int_{0}^{1} G(s, s) a(s) d s+\frac{|\alpha|}{|1-\alpha|} \int_{0}^{1} G(\eta, s) a(s) d s<1 .
$$

Then the boundary value problem (1.1), (1.2) has at least one nontrivial symmetric solution $u^{*} \in C([0,1])$.

Proof. Let

$$
A=\int_{0}^{1} G(s, s) a(s) d s+\frac{|\alpha|}{|1-\alpha|} \int_{0}^{1} G(\eta, s) a(s) d s
$$

and

$$
B=\int_{0}^{1} G(s, s) b(s) d s+\frac{|\alpha|}{|1-\alpha|} \int_{0}^{1} G(\eta, s) b(s) d s .
$$

Then we have $A<1$. Since $f(t, 0) \not \equiv 0$, there exists a subinterval $[\sigma, \tau] \subset[0,1]$ such that

$$
\min _{\sigma \leq t \leq \tau}|f(t, 0)|>0 \text {. }
$$


On the other hand, the inequality $b(t) \geq|f(t, 0)|$ holds for a. e. $t \in[0,1]$ and thus we know that $B>0$. Put $r=B(1-A)^{-1}$ and

$$
\Omega=\{u \in C([0,1]): u \text { is symmetric on }[0,1] \text { and }\|u\|<r\} .
$$

Suppose that $u \in \partial \Omega$ and $\lambda>1$ are such that $T u=\lambda u$. Then

$$
\begin{aligned}
\lambda r= & \lambda\|u\|=\|T u\|=\max _{0 \leq t \leq 1}|(T u)(t)| \\
\leq & \int_{0}^{1} G(s, s)|f(s, u(s))| d s+\frac{|\alpha|}{|1-\alpha|} \int_{0}^{1} G(\eta, s)|f(s, u(s))| d s \\
\leq & \int_{0}^{1} G(s, s)(a(s)|u(s)|+b(s)) d s \\
& +\frac{|\alpha|}{|1-\alpha|} \int_{0}^{1} G(\eta, s)(a(s)|u(s)|+b(s)) d s \\
\leq & \left(\int_{0}^{1} G(s, s) a(s) d s+\frac{|\alpha|}{|1-\alpha|} \int_{0}^{1} G(\eta, s) a(s) d s\right)\|u\| \\
& +\int_{0}^{1} G(s, s) b(s) d s+\frac{|\alpha|}{|1-\alpha|} \int_{0}^{1} G(\eta, s) b(s) d s \\
= & A\|u\|+B=A r+B .
\end{aligned}
$$

Therefore,

$$
\lambda \leq A+\frac{B}{r}=A+\frac{B}{B(1-A)^{-1}}=A+(1-A)=1,
$$

which contradicts the inequality $\lambda>1$. By Theorem 1.1, $T$ has a fixed point $u^{*} \in \bar{\Omega}$. By $f(t, 0) \not \equiv 0$, the boundary value problem (1.1), (1.2) has a nontrivial symmetric solution $u^{*} \in C([0,1])$. This completes the proof.

Theorem 2.2. Suppose that $f \in C([0,1] \times \mathbb{R}, \mathbb{R}), f(t, 0) \not \equiv 0, \alpha \neq 1$, and there exist two non-negative symmetric functions $a, b \in L^{1}[0,1]$ such that

$$
|f(t, x)| \leq a(t)|x|+b(t) \quad \text { for a. e. } t \in[0,1] \text { and all } x \in \mathbb{R}
$$

Assume that one of the following hypotheses is satisfied:

(1) There exists a $p>1$ such that $a \in L^{p}[0,1]$ and

$$
\int_{0}^{1} a^{p}(s) d s<\left(\frac{4|1-\alpha|(1+q)^{1 / q}}{|1-\alpha|(1+q)^{1 / q}+4|\alpha| \eta(1-\eta)}\right)^{p},
$$

where $1 / p+1 / q=1$.

(2) There exists a constant $\mu>-1$ such that

$$
a(s) \leq \frac{4^{1+\mu}|1-\alpha|}{|1-\alpha|+|\alpha|}[s(1-s)]^{\mu} \quad \text { for a.e. } s \in[0,1] .
$$


(3) The function a satisfies

$$
a(s) \leq \frac{6|1-\alpha|}{|1-\alpha|+3|\alpha| \eta(1-\eta)} \quad \text { for a.e. } s \in[0,1]
$$

and

$$
\operatorname{mes}\left\{s \in[0,1]: a(s)<\frac{6|1-\alpha|}{|1-\alpha|+3|\alpha| \eta(1-\eta)}\right\}>0 \text {. }
$$

Then the boundary value problem (1.1), (1.2) has at least one nontrivial solution $u^{*} \in C([0,1])$.

Proof. Let $A$ be given by formula (2.5). In order to apply Theorem 2.1, we only need to prove that $A<1$.

(1) By using the Hölder inequality, we have

$$
\begin{aligned}
A & \leq\left(\int_{0}^{1} a^{p}(s) d s\right)^{1 / p}\left\{\left(\int_{0}^{1}[G(s, s)]^{q} d s\right)^{1 / q}+\frac{|\alpha|}{|1-\alpha|}\left(\int_{0}^{1}[G(\eta, s)]^{q} d s\right)^{1 / q}\right\} \\
& \leq\left(\int_{0}^{1} a^{p}(s) d s\right)^{1 / p}\left(\frac{1}{4}+\frac{|\alpha|}{|1-\alpha|} \frac{\eta(1-\eta)}{(1+q)^{1 / q}}\right) \\
& <\frac{4|1-\alpha|(1+q)^{1 / q}}{|1-\alpha|(1+q)^{1 / q}+4|\alpha| \eta(1-\eta)} \frac{|1-\alpha|(1+q)^{1 / q}+4|\alpha| \eta(1-\eta)}{4|1-\alpha|(1+q)^{1 / q}}=1 .
\end{aligned}
$$

(2) In this case, we have

$$
\begin{aligned}
A & \leq \frac{4^{1+\mu}|1-\alpha|}{|1-\alpha|+|\alpha|}\left(\int_{0}^{1} G(s, s)[s(1-s)]^{\mu} d s+\frac{|\alpha|}{|1-\alpha|} \int_{0}^{1} G(\eta, s)[s(1-s)]^{\mu} d s\right) \\
& \leq \frac{4^{1+\mu}|1-\alpha|}{|1-\alpha|+|\alpha|}\left(\int_{0}^{1}[s(1-s)]^{1+\mu} d s+\frac{|\alpha|}{|1-\alpha|} \int_{0}^{1} G(s, s)[s(1-s)]^{\mu} d s\right) \\
& <\frac{4^{1+\mu}|1-\alpha|}{|1-\alpha|+|\alpha|}\left(\frac{1}{4^{1+\mu}}+\frac{|\alpha|}{|1-\alpha|} \frac{1}{4^{1+\mu}}\right) \\
& =\frac{4^{1+\mu}|1-\alpha|}{|1-\alpha|+|\alpha|} \frac{|1-\alpha|+|\alpha|}{4^{1+\mu}|1-\alpha|}=1 .
\end{aligned}
$$

(3) In this case, we have

$$
\begin{aligned}
A & <\frac{6|1-\alpha|}{|1-\alpha|+3|\alpha| \eta(1-\eta)}\left(\int_{0}^{1} G(s, s) d s+\frac{|\alpha|}{|1-\alpha|} \int_{0}^{1} G(\eta, s) d s\right) \\
& =\frac{6|1-\alpha|}{|1-\alpha|+3|\alpha| \eta(1-\eta)}\left(\frac{1}{6}+\frac{|\alpha| \eta(1-\eta)}{2|1-\alpha|}\right)=1 .
\end{aligned}
$$

The proof is complete. 
Corollary 2.1. Suppose that $f \in C([0,1] \times \mathbb{R}, \mathbb{R}), f(t, 0) \not \equiv 0, \alpha \neq 1$, and

$$
\Delta:=\limsup _{|x| \rightarrow \infty} \max _{t \in[0,1]}\left|\frac{f(t, x)}{x}\right|<\frac{6|1-\alpha|}{|1-\alpha|+3|\alpha| \eta(1-\eta)} .
$$

Then problem (1.1), (1.2) has at least one nontrivial solution $u^{*} \in C([0,1])$.

Proof. Let us put $\varepsilon=\frac{1}{2}\left(\frac{6|1-\alpha|}{|1-\alpha|+3|\alpha| \eta(1-\eta)}-\Delta\right)$. Then there exists $c>0$ such that

$$
|f(t, x)| \leq\left(\frac{6|1-\alpha|}{|1-\alpha|+3|\alpha| \eta(1-\eta)}-\varepsilon\right)|x|
$$

for $(t, x) \in[0,1] \times \mathbb{R} \backslash(-c, c)$. Set $M=\max \{|f(t, x)|:(t, x) \in[0,1] \times[-c, c]\}$. Then

$$
|f(t, x)| \leq\left(\frac{6|1-\alpha|}{|1-\alpha|+3|\alpha| \eta(1-\eta)}-\varepsilon\right)|x|+M
$$

for $(t, x) \in[0,1] \times \mathbb{R}$. Consequently, the assumption of Theorem 2.2(3) is satisfied, where

$$
a(s)=\frac{6|1-\alpha|}{|1-\alpha|+3|\alpha| \eta(1-\eta)}-\varepsilon
$$

and $b(s)=M$ for $s \in[0,1]$.

Corollary 2.2. Suppose that $f \in C([0,1] \times \mathbb{R}, \mathbb{R}), f(t, 0) \not \equiv 0, \alpha \in(0,1)$, and there exist two non-negative symmetric functions $a, b \in L^{1}[0,1]$ such that

$$
|f(t, x)| \leq a(t)|x|+b(t) \quad \text { for a. e. } t \in[0,1] \text { and all } x \in \mathbb{R} .
$$

Let one of following assumptions hold:

(1) There exists constant $p>1$ such that $a \in L^{p}[0,1]$ and

$$
\int_{0}^{1} a^{p}(s) d s<(4(1-\alpha))^{p} .
$$

(2) There exists constant $\mu>-1$ such that

$$
a(s) \leq 4^{1+\mu}(1-\alpha)(s(1-s))^{\mu} \quad \text { for a. e. } s \in[0,1] .
$$

(3) The function a satisfies the estimate

$$
a(s) \leq 6(1-\alpha) \quad \text { for a.e. } s \in[0,1] .
$$

Then the boundary value problem (1.1), (1.2) has at least one nontrivial solution $u^{*} \in C([0,1])$.

Proof. The validity of the corollary follows immediately from Theorem 2.2 by using the inequality $\eta(1-\eta) \leq \frac{1}{4}$. 
Corollary 2.3. Suppose that $f \in C([0,1] \times \mathbb{R}, \mathbb{R}), f(t, 0) \not \equiv 0, \alpha \in(0,1)$, and

$$
\Lambda:=\limsup _{|x| \rightarrow \infty} \max _{t \in[0,1]}\left|\frac{f(t, x)}{x}\right| \leq 6(1-\alpha) .
$$

Then the boundary value problem (1.1), (1.2) has at least one nontrivial solution $u^{*} \in C([0,1])$.

Proof. The validity of the corollary follows immediately from Corollary 2.1 by using the inequality $\eta(1-\eta) \leq \frac{1}{4}$.

\section{EXAMPLES}

In this section, in order to illustrate the results obtained, we consider some examples.

Example 3.1. Consider the three-point boundary value problem

$$
\begin{gathered}
u^{\prime \prime}+\min \{t, 1-t\} u(5 \sin u-7)+\sin (t(1-t))=0, \quad 0<t<1, \\
u(0)=u(1)=-u(1 / 2) .
\end{gathered}
$$

Set $\alpha=-1, \eta=\frac{1}{2}, f(t, x)=\min \{t, 1-t\} x(5 \sin x-7)+\sin [t(1-t)]$ for $(t, x) \in$ $[0,1] \times \mathbb{R}$, and $a(t)=12 \min \{t, 1-t\}$ and $b(t)=\sin [t(1-t)]$ for $t \in[0,1]$. It is easy to see that $a, b \in L^{1}[0,1]$ are non-negative, symmetric, and the inequality $|f(t, x)| \leq$ $a(t)|x|+b(t)$ holds for all $(t, x) \in[0,1] \times \mathbb{R}$. Moreover, we have

$$
A=\int_{0}^{1} G(s, s) a(s) d s+\frac{|\alpha|}{|1-\alpha|} \int_{0}^{1} G(\eta, s) a(s) d s=\frac{5}{8}+\frac{1}{4}<1 .
$$

Hence, by Theorem 2.1, the boundary value problem (3.1) has at least one nontrivial symmetric solution.

Example 3.2. Consider the three-point boundary value problem

$$
\begin{gathered}
u^{\prime \prime}+\frac{2 \sqrt{6 t(1-t)}}{1+u^{4}} u^{5} e^{-u^{2}}+5 e^{t(1-t)}=0, \quad 0<t<1, \\
u(0)=u(1)=2 u(1 / 4) .
\end{gathered}
$$

Set $\alpha=2, \eta=\frac{1}{4}, f(t, x)=\frac{2 \sqrt{6 t(1-t)}}{1+x^{4}} x^{5} e^{-x^{2}}+5 e^{t(1-t)}$ for $(t, x) \in[0,1] \times \mathbb{R}$, and $a(t)=2 \sqrt{6 t(1-t)}$ and $b(t)=5 e^{t(1-t)}$ for $t \in[0,1]$. It is easy to prove that $a, b \in$ $L^{1}[0,1]$ are non-negative, symmetric, and the inequality $|f(t, x)| \leq a(t)|x|+b(t)$ holds for all $(t, x) \in[0,1] \times \mathbb{R}$. If we put $p=q=2$, then

$$
\int_{0}^{1} a^{p}(s) d s=\int_{0}^{1} 24 s(1-s) d s=4,
$$

and

$$
\left(\frac{4|1-\alpha|(1+q)^{1 / q}}{|1-\alpha|(1+q)^{1 / q}+4|\alpha| \eta(1-\eta)}\right)^{p}=64(7-4 \sqrt{3}) \text {. }
$$


Therefore

$$
\int_{0}^{1} a^{p}(s) d s<\left(\frac{4|1-\alpha|(1+q)^{1 / q}}{|1-\alpha|(1+q)^{1 / q}+4|\alpha| \eta(1-\eta)}\right)^{p} .
$$

Hence, by Theorem 2.2(1), the boundary value problem (3.2) has at least one nontrivial symmetric solution.

Example 3.3. Consider the three-point boundary value problem

$$
\begin{gathered}
u^{\prime \prime}+\frac{4 u^{3} \sqrt{t(1-t)}}{5\left(1+u^{2}\right)}+4 t(1-t) u-\max \{t, 1-t\}=0, \quad 0<t<1, \\
u(0)=u(1)=-2 u(\eta) .
\end{gathered}
$$

Set $\alpha=-2, \eta \in(0,1), f(t, x)=\frac{4 x^{3} \sqrt{t(1-t)}}{5\left(1+x^{2}\right)}+4 t(1-t) x-\max \{t, 1-t\}$ for $(t, x) \in$ $[0,1] \times \mathbb{R}$, and $a(t)=\frac{4}{5} \sqrt{t(1-t)}+4 t(1-t)$ and $b(t)=\max \{t, 1-t\}$ for $t \in[0,1]$. It is easy to prove that $a, b \in L^{1}[0,1]$ are non-negative, symmetric, and the inequality $|f(t, x)| \leq a(t)|x|+b(t)$ holds for all $(t, x) \in[0,1] \times \mathbb{R}$. If we put $\mu=\frac{1}{2}$, then

$$
\begin{aligned}
a(s) & =\frac{4}{5} \sqrt{s(1-s)}+4 s(1-s) \leq \frac{24}{5} \sqrt{s(1-s)} \\
& =\frac{4^{1+\mu}|1-\alpha|}{|1-\alpha|+|\alpha|}[s(1-s)]^{\mu}
\end{aligned}
$$

for $s \in[0,1]$. Hence, by Theorem 2.2(2), the boundary value problem (3.3) has at least one nontrivial symmetric solution.

\section{REFERENCES}

[1] K. Deimling, Nonlinear functional analysis. Berlin: Springer-Verlag, 1985.

[2] W. Feng and J. R. L. Webb, "Solvability of $m$-point boundary value problems with nonlinear growth," J. Math. Anal. Appl., vol. 212, no. 2, pp. 467-480, 1997.

[3] W. Feng and J. R. L. Webb, "Solvability of three point boundary value problems at resonance," in Proceedings of the Second World Congress of Nonlinear Analysts, Part 6 (Athens, 1996), vol. 30, no. 6, 1997, pp. 3227-3238.

[4] C. P. Gupta, "Solvability of a three-point nonlinear boundary value problem for a second order ordinary differential equation," J. Math. Anal. Appl., vol. 168, no. 2, pp. 540-551, 1992.

[5] C. P. Gupta, S. K. Ntouyas, and P. C. Tsamatos, "Solvability of an $m$-point boundary value problem for second order ordinary differential equations," J. Math. Anal. Appl., vol. 189, no. 2, pp. 575584, 1995.

[6] C. P. Gupta, S. K. Ntouyas, and P. C. Tsamatos, "Solvability of an $m$-point boundary value problem for second order ordinary differential equations," J. Math. Anal. Appl., vol. 189, no. 2, pp. 575584, 1995.

[7] C. P. Gupta and S. I. Trofimchuk, "A sharper condition for the solvability of a three-point second order boundary value problem," J. Math. Anal. Appl., vol. 205, no. 2, pp. 586-597, 1997.

[8] V. A. Il'in and E. I. Moiseev, "A nonlocal boundary value problem of the second kind for the Sturm-Liouville operator," Differentsial'nye Uravneniya, vol. 23, no. 8, pp. 1422-1431, 1471, 1987. 
[9] G. Infante and J. R. L. Webb, "Nonzero solutions of Hammerstein integral equations with discontinuous kernels," J. Math. Anal. Appl., vol. 272, no. 1, pp. 30-42, 2002.

[10] N. Kosmatov, "Symmetric solutions of a multi-point boundary value problem," J. Math. Anal. Appl., vol. 309, no. 1, pp. 25-36, 2005.

[11] B. Liu, "Positive solutions of a nonlinear three-point boundary value problem," Comput. Math. Appl., vol. 44, no. 1-2, pp. 201-211, 2002.

[12] B. Liu, "Positive solutions of a nonlinear three-point boundary value problem," Appl. Math. Comput., vol. 132, no. 1, pp. 11-28, 2002.

[13] R. Ma, "Positive solutions of a nonlinear three-point boundary-value problem," Electron. J. Differential Equations, pp. No. 34, 8 pp. (electronic), 1999.

[14] R. Ma, "Multiplicity of positive solutions for second-order three-point boundary value problems," Comput. Math. Appl., vol. 40, no. 2-3, pp. 193-204, 2000.

[15] R. Ma, "Positive solutions for second-order three-point boundary value problems," Appl. Math. Lett., vol. 14, no. 1, pp. 1-5, 2001.

[16] R. Ma, "Positive solutions of a nonlinear m-point boundary value problem," Comput. Math. Appl., vol. 42, no. 6-7, pp. 755-765, 2001

[17] Y. H. Ma and R. Y. Ma, "Positive solutions of a singular nonlinear three-point boundary value problem," Acta Math. Sci. Ser. A Chin. Ed., vol. 23, no. 5, pp. 583-588, 2003.

[18] Y. Sun, "Eigenvalues and symmetric positive solutions for a three-point boundary-value problem," Electron. J. Differential Equations, pp. No. 127, 7 pp. (electronic), 2005.

[19] Y. Sun and L. Liu, "Solvability for a nonlinear second-order three-point boundary value problem," J. Math. Anal. Appl., vol. 296, no. 1, pp. 265-275, 2004.

\section{Author's address}

\section{Yongping Sun} China

Department of Fundamental Courses, Hangzhou Radio \& TV University, Hangzhou, Zhejiang 310012,

E-mail address: sunyongping@126.com 